\title{
NON-EQUILIBRIUM DYNAMICS OF HOT ABELIAN HIGGS MODEL
}

\author{
A. RAJANTIE \\ Centre for Theoretical Physics, \\ University of Sussex, \\ Brighton BN1 9QH, \\ United Kingdom \\ E-mail: a.k.rajantie@sussex.ac.uk
}

\begin{abstract}
The real-time dynamics of finite-temperature gauge theories can be approximated, to leading-order accuracy in the coupling constants, by a classical field theory with the hard thermal loop Lagrangian. I show how this approach can be used in numerical lattice simulations to study dynamics of the Abelian Higgs model in or slightly out of equilibrium.
\end{abstract}

\section{Hard thermal loops}

Depending on its details, the electroweak phase transition may explain the observed baryon asymmetry in the Universe.t Although static equilibrium properties such as the phase structure of the theory have been determined to high accuracy with numerical lattice simulations, 1 much less is known about non-equilibrium dynamics, or even real-time correlators in equilibrium. The reason is that to calculate any real-time correlator, one needs to evaluate a Minkowskian path integral, which cannot be done using Monte Carlo methods, and perturbation theory breaks down because of infrared problems.

Assuming that the fields are initially in thermal equilibrium, the occupation number of the soft modes (with pomentum $k \lesssim g T$ ) is large, and they can be approximated by classical fields 3 The hard modes $(k \gtrsim T)$ have a small occupation number and must be treated as quantum fields, but perturbation theory works well for them, and they can be integrated out perturbatively. This is done by choosing a lattice cutoff $\delta x=1 / \Lambda$ with $g T \ll \Lambda \ll T$, calculating one-loop correlators both in the full theory and in the lattice theory and determining the effective Lagrangian by matching the results. Because $\Lambda$ acts as an infrared cutoff, this construction is free from infrared problems, and the expansion parameter is $g^{2}$, not $g^{2} T / m$ as usually is the case in perturbative calculations. In practice, since the full lattice result 1 is very cumbersome and therefore unsuitable for numerical simulations, it is easier to match only static correlators, which gives a small error to the results.

In this way, an effective theory can be constructed for the soft modes, and

wscosmo: submitted to World Scientific on October 27, 2018 
the dynamics of this effective theory is well described by classical equations of motion. This approach has previously been used to measure the sphaleron rate of hot $\mathrm{SU}(2)$ gauge theory. $\mathrm{G}$ I will argue that it can also be used for simulating non-equilibrium dynamics of phase transitions.

In the case of the Abelian Higgs model, the effective Lagrangian is

$$
\begin{aligned}
\mathcal{L}_{\mathrm{HTL}}= & -\frac{1}{4} F_{\mu \nu} F^{\mu \nu}-\frac{1}{4} m_{D}^{2} \int \frac{d \Omega}{4 \pi} F^{\mu \alpha} \frac{v_{\alpha} v^{\beta}}{(v \cdot \partial)^{2}} F_{\mu \beta} \\
& +\left(D_{\mu} \phi\right)^{*} D^{\mu} \phi-m_{T}^{2} \phi^{*} \phi-\lambda\left(\phi^{*} \phi\right)^{2}
\end{aligned}
$$

where $m_{D}^{2}=\frac{1}{3} e^{2} T^{2}-\delta m_{D}^{2}$ and $m_{T}^{2}=m^{2}+\left(e^{2} / 4+\lambda / 3\right) T^{2}-\delta m_{T}^{2}$. The mass counterterms are $\delta m_{D}^{2}=e^{2} \Sigma T / 4 \pi \delta x$ and $\delta m_{T}^{2}=\left(3 e^{2}+4 \lambda\right) \Sigma T / 4 \pi \delta x$. The integration is taken over the unit sphere of velocities $v=(1, \vec{v}), \vec{v}^{2}=1$. The extra terms in the Lagrangian (1D) depend only on the couplings and the temperature, as long as the high-temperature approximation $T \gg m$ is valid.

The equations of motion can be derived from Eq. (1), and are

$$
\begin{aligned}
\partial_{\mu} F^{\mu \nu} & =m_{D}^{2} \int \frac{d \Omega}{4 \pi} \frac{v^{\nu} v^{i}}{v \cdot \partial} E^{i}-2 e \operatorname{Im} \phi^{*} D^{\nu} \phi, \\
D_{\mu} D^{\mu} \phi & =-m_{T}^{2} \phi-2 \lambda\left(\phi^{*} \phi\right) \phi .
\end{aligned}
$$

Because of the derivative in the denominator, the gauge field equation of motion is non-local.

\section{Local formulation}

To make numerical simulations feasible, one needs a local formulation for the theory. The most straightforward approaches invglve describing the hard modes by a large number of charged point particles, or by the phase-space distribution of these particles.6. 6 In practice, both formulations lead to a 5+1dimensional field theory. However, in the Abelian case, one can integrate out one of the dimensions, thus obtaining a 4+1-dimensional theory that is completely equivalent with the others. $\theta(t, \vec{x}, z)$, where $z \in[0,1]$. In the temporal gauge, they satisfy the equations of motion

$$
\begin{aligned}
\partial_{0}^{2} \vec{f}(z) & =z^{2} \vec{\nabla}^{2} \vec{f}+m_{D} z \sqrt{\frac{1-z^{2}}{2}} \vec{\nabla} \times \vec{A}, \\
\partial_{0}^{2} \theta(z) & =z^{2} \vec{\nabla} \cdot\left(\vec{\nabla} \theta-m_{D} \vec{A}\right) \\
\partial_{0}^{2} \vec{A} & =-\vec{\nabla} \times \vec{\nabla} \times \vec{A}-2 e \operatorname{Im} \phi^{*} \vec{D} \phi
\end{aligned}
$$

wscosmo: submitted to World Scientific on October 27, 2018 


$$
+m_{D} \int_{0}^{1} d z z^{2}\left(\vec{\nabla} \theta-m_{D} \vec{A}+\sqrt{\frac{1-z^{2}}{2 z^{2}}} \vec{\nabla} \times \vec{f}\right) .
$$

With these equations, it is possible to calculate any real-time correlator at finite temperature. One simply takes a large number of initial configurations from the thermalensemble with the probability distribution $\exp (-\beta H)$, using the Hamiltonian corresponding to the equations of motion (3), and evolves each configuration in time, measuring the correlator of interest. The average over the initial configurations gives the ensemble average of the correlator.

\section{Simulations}

In addition to the standard lattice discretization, the dependence on the new coordinate $z$ needs to be discretized as well. $\mathrm{A}$ convenient way is to define the canonical momenta $\vec{F}=\partial_{0} \vec{f}$ and $\Pi=\partial_{0} \theta$ and to expand both the fields and the momenta in terms of Legendre polynomials:

$$
\begin{array}{ll}
\vec{f}^{(n)}=\int_{0}^{1} d z z \sqrt{\frac{2}{1-z^{2}}} P_{2 n}(z) \vec{f}(z), & \theta^{(n)}=\int_{0}^{1} d z P_{2 n}(z) \theta(z), \\
\vec{F}^{(n)}=\int_{0}^{1} \frac{d z}{z} \sqrt{\frac{2}{1-z^{2}}} P_{2 n}(z) \vec{F}(z), & \Pi^{(n)}=\int_{0}^{1} d z P_{2 n}(z) \Pi(z) .
\end{array}
$$

The Hamiltonian can be written in terms of these Legendre modes, and the ensemble of initial configurations can be generated using standard Monte Carlo techniques. This can be done in two steps:

(i) The Hamiltonian is Gaussian in the hard fields $\vec{f}, \vec{F}, \theta$ and $\Pi$, and they can therefore be integrated out analytically. This leads to the Hamiltonian of the ordinary classical Abelian Higgs model, with an extra Debye screening term for the electric field. This classical Hamiltonian can be used to generate the initial configuration for the soft modes.

(ii) Given the soft configuration from step (i), the hard field configuration can be generated very effectively, since the hard Hamiltonian is Gaussian.

After the initial configurations have been generated, any real-time correlator can be measured as was explained in the end of Sec. 2 .

\section{Non-equilibrium dynamics}

In addition to equilibrium real-time correlators, the formulation presented here can also be used to study non-equilibrium dynamics, provided that the

wscosmo: submitted to World Scientific on October 27, 2018 
hard modes remain close enough to the equilibrium. This is plausible in a phase transition, since the phase of a system is a property of the longwavelength modes only. Near the phase transition, in both phases, all the masses are suppressed by powers of the coupling constant $g$ relative to the temperature, and the high-temperature approximation can be used. In this approximation, the phase of the system does not enter the results of the oneloop diagrams, i.e. the distribution of the hard modes is indeed the same in both phases, within the accuracy of our approach. Thus, there is no reason for the hard modes to fall out of equilibrium during the transition.

A natural way of studying a phase transition would be to start from thermal equilibrium in the Coulomb phase, and decrease the temperature so that the system undergoes a transition to the Higgs phase. This requires a mechanism for changing the temperature, and in practice, it is easier to keep the temperature constant and change the parameters, such as the mass of the Higgs field, instead. In fact, when $\lambda \ll e^{2}$, even that is not necessary. The transition is of first order, and if one thermalizes the system initially to the metastable Coulomb phase below $T_{c}$, bubbles of the Higgs phase nucleate during the time evolution, and the phase transition takes place. Assuming that the latent heat is small enough, the temperature does not change significantly. In this way, many non-equilibrium properties of the phase transition can be studied non-perturbatively.

\section{Acknowledgments}

I would like to thank M. Hindmarsh for collaboration on this topic.

\section{References}

1. V. A. Rubakov and M. E. Shaposhnikov, Usp. Fiz. Nauk 166, 493 (1996).

2. K. Kajantie et al., Nucl. Phys. B 493, 413 (1997).

3. D. Y. Grigoriev and V. A. Rubakov, Nucl. Phys. B 299, 67 (1988).

4. B. J. Nauta, hep-ph/9906389.

5. C. R. Hu and B. Müller, Phys. Lett. B 409, 377 (1997);

G. D. Moore, C. Hu, and B. Müller, Phys. Rev. D 58, 045001 (1998).

6. D. Bödeker, G. D. Moore and K. Rummukainen, hep-ph/9907545.

7. A. Rajantie and M. Hindmarsh, Phys. Rev. D 60, 096001 (1999).

8. M. Laine and A. Rajantie, Nucl. Phys. B 513, 471 (1998).

9. E. Iancu, Phys. Lett. B 435, 152 (1998); hep-ph/9809535.

wscosmo: submitted to World Scientific on October 27, 2018 